\title{
Downhole Mechanical Source Design and Its Reliability Analysis Based on Finite Element Method
}

\author{
Gang LIU ${ }^{1, a}$, Hang WANG ${ }^{1, b}$ and Jiayang WANG ${ }^{2, c}$ \\ ${ }^{1}$ Chian university of petroleum, Qingdao ShandongChina \\ 2 Shandong Kerui Petroleum Equipment Co. Ltd, Dongying Shandong China \\ aemail:Ig87323@126.com, bemail:wanghang@s.upc.edu.cn, cemail:1326737095@qq.com
}

Keywords: Downhole vibration source, Structure design, Reliability analysis.

\begin{abstract}
In the directional well anti-collision ground monitoring system, with the well depth increases, the ground monitoring signal intensity decreases, which can not meet the requirements of monitoring. A mechanical downhole source capable of providing a certain strength standard signal and capable of resuming vibrations is designed, which mainly comprises four parts: a lifting device, a torque transmission device, a down strike device and a sealing device. The key locking mechanism of the system was analyzed. The relation between the parameters of the sliding body and the strength and the release force of the mechanism was obtained. The mechanism was verified to regulate the release force in a wide range and satisfy the strength design requirements. Finally, the fault tree model of "failure of source underground" was established, the reliability of downhole source was analyzed, and control measures were put forward according to the possible damage modes.
\end{abstract}

\section{基于有限元的井下机械震源设计及其可靠性分析}

\author{
刘刚 1 , a , 王航 ${ }^{1, b}$, 汪嘉洋 $2, c$ \\ 1中国石油大学 (华东), 青岛, 山东, 中国 \\ ${ }^{2}$ 山东科瑞石油装备有限公司, 东营, 山东, 中国
}

aemail: Ig87323@126.com, bemail:wanghang@s.upc.edu.cn, cemail: 1326737095@qq.com

关键词：井下震源；结构设计；可靠性分析

中文摘要. 在定向井防碰地面监测系统中, 随着井深的增加, 地面监测信号强度降低, 不能 满足监测要求。设计了一种能够提供一定强度标准信号且可重复起振的机械式井下震源, 主 要包括：上提装置、扭矩传递装置、下击装置和密封装置四个部分。并对系统关键的锁紧机 构进行技术分析, 得到卡瓦体参数与机构强度及释放力的关系, 验证该机构可在较大的范围 内调节释放力, 且满足强度设计要求。最后建立了 “井下震源失效” 故障树, 对井下震源进 行可靠性分析，针对可能出现的损坏模式提出控制措施。

1. 引言

老井网的加密调整可以提高老油田的采收率，但在加密调整井钻井过程中，存在井眼碰 撞风险。针对从式井网整体加密调整的井眼防碰问题, 可以通过对钻头运动产生的振动信号 进行监测, 确保钻头与邻井套管碰撞之前进行报警, 避免加密钻井时对邻井套管产生碰撞, 
保障钻井作业安全 ${ }^{[1,2]}$ 。钻头破碎岩石时会产生振动, 但是这种震源信号强度不足、信号不标 准导致后期分析困难 ${ }^{[3-5]}$ 。为满足井间距离监测的需要。亟需设计一种能够提供一定强度标准 信号且能够重复起震的井下震源, 以实现钻头与风险邻井之间相对位置的预测。本文研制的 机械式井下震源可以满足上述要求。

\section{2. 井下机械震源结构与功能实现}

\section{1 井下机械震源结构}

本文所设计的井下机械震源整体结构如图1所示，整个震源包括：下击装置、上提装置、 扭矩传递装置及密封装置四个部分。

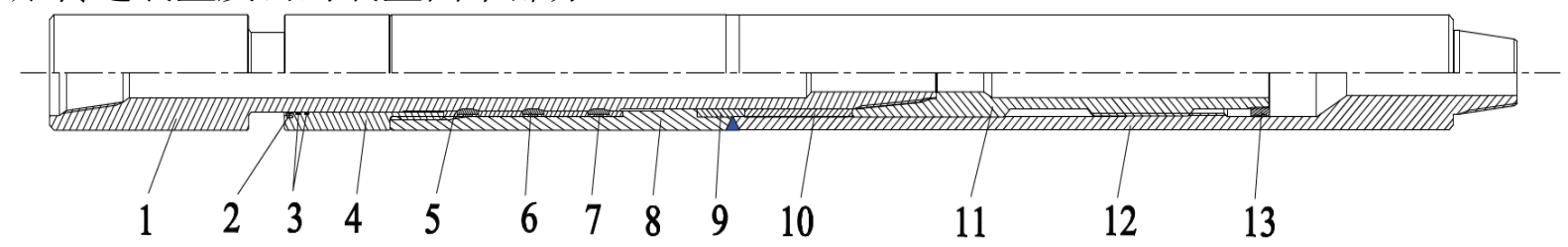

1一上心轴； 2 - $\mathrm{A}$ 型防尘圈； 3 -0 型密封圈； 4一打击套筒； 5一圆筒形卡瓦座； 6一卡瓦体； 7一弹性体；8一中筒体；9一短隔套；10一长隔套；11一下心轴；12一下筒体；13一活塞环

图1＼cjkstart井下机械震源

下击装置作用是为系统的重复起震产生下击力, 见图2。锁紧机构是由上心轴、中筒体、 卡瓦体、圆筒形卡瓦座以及弹性体组成, 见图3。其中上心轴上端加工螺纹, 用于和上部钻具 进行连接。锁紧机构主要由圆筒形卡瓦座以及安装在上面的 24 个卡瓦体组成。在圆筒形卡瓦 座上, 从 3 个不同的横截面分别开 8 个周向均布的槽, 安装并约束 24 个卡瓦体的径向运动、轴 向运动。在震源工作的过程中, 卡瓦体在接触力作用下实现上心轴的锁紧与解锁, 控制上心 轴的下行，撞击打击套筒，产生震动。

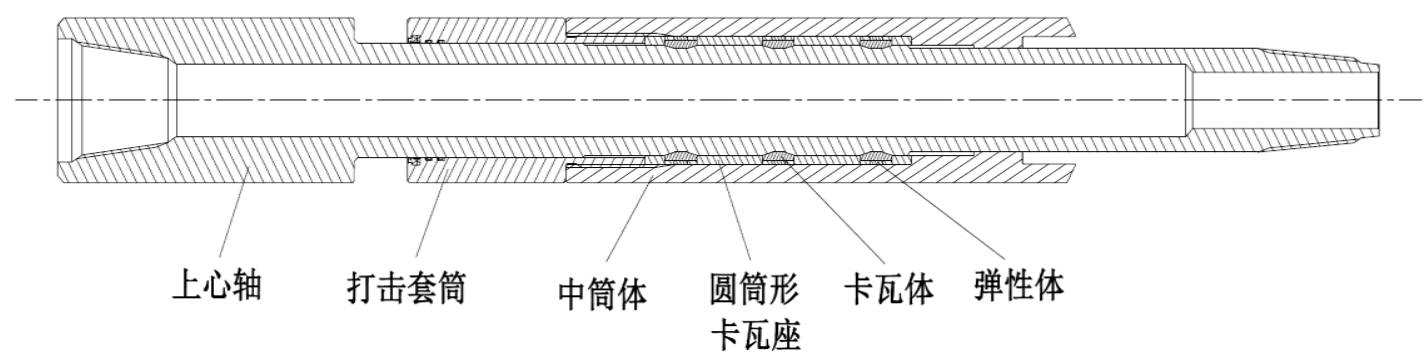

图2 下击装置

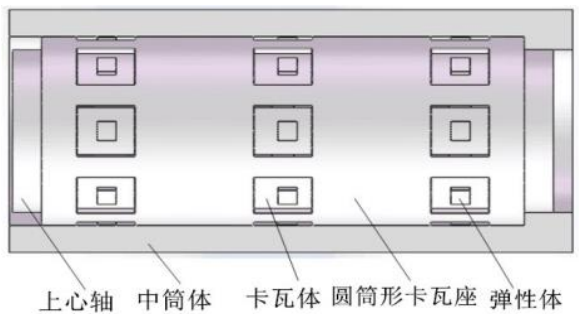

图3 锁紧机构

上提装置的作用是传递心轴与筒体间的轴向力。短隔套、长隔套可以提供液压大钳工作 空间, 方便上心轴与下心轴的螺纹联接, 并且可以进行轴向约束, 传递轴向力。扭矩传递装 置的作用是传递心轴与筒体间的扭矩。下心轴加工外花键结构, 下筒体加工内花键结构, 二 者通过花键联接, 承受螺杆钻具的反扭矩。合理的密封结构和优质密封材料的选用可以阻止 内部润滑油的泄漏及外部钻井液的渗入 ${ }^{[6]}$ 。密封装置包括4组结构。分别为A型防尘圈加一对 $\mathrm{O}$ 型密封圈密封方式、端面密封方式、钻杆螺纹密封方式以及台肩端面密封方式。 


\section{2 功能实现}

该井下震源系统的设计, 满足其与井下钻具的连接、重复起震以及旋转钻进这三个功能。 其工作流程如图4所示。

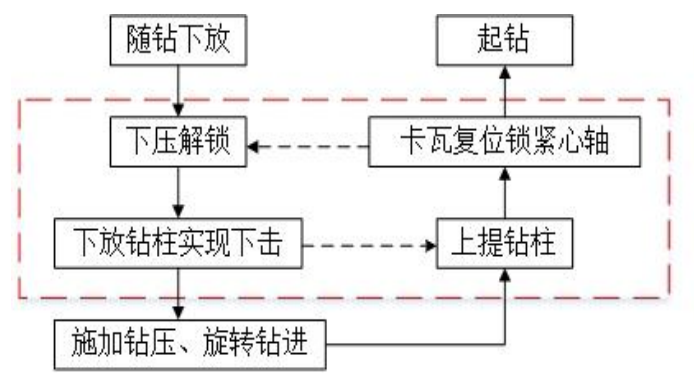

图 4 井下机械震源工作过程示意图

连接：机械震源通过上下心轴上的螺纹与上下部钻具进行连接。

重复起震：卡瓦体的受力如图5所示。当上部钻具未施加钻压时, 卡瓦体主要受到卡瓦座 的约束力F1、f1、F2、f2作用, 使得 24 个卡瓦体产生对上心轴的接触力N1, 此时上心轴被卡 瓦体锁紧; 随着上部钻具施加钻压, 上心轴受到的轴向压力增大, 使得卡瓦体受到上心轴的 接触力N1增大。当N1水平方向上的作用力大于圆筒形卡瓦座对卡瓦体的水平约束力 F1、 $\mathrm{f} 1$ 、 F2、f2时, 卡瓦体就会产生变形及径向运动, 机构解锁, 进而上心轴下行并撞击打击套筒, 产生震动, 得到一次标准信号。重复起震时, 上提钻柱。上心轴跟随钻柱向上运动, N1随之 减小。卡瓦体在弹性体的法向力Fk及圆筒形卡瓦座约束力F1、f1、F2、f2的共同作用下，恢 复变形。当Fk大于 F1、f1、F2、f2的水平方向作用力时, 卡瓦体产生径向运动, 上心轴再次 受到卡瓦体的接触力N1，最终锁紧机构实现锁紧。

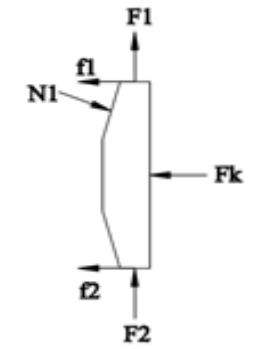

图5 卡瓦体受力示意图

旋转钻进: 在进行旋转钻进时, 该机械震源可通过下心轴与下筒体之间的花键结构承受螺 杆钻具的反扭矩, 实现钻进功能。

\section{3. 关键技术分析}

为保障震源实现 “能够提供一定强度的标准信号且能够重复起震” 的功能, 需要对关键 的锁紧机构部件进行技术分析。卡瓦体结构是实现重复起震功能的重要部件之一。通过设计 不同的结构参数, 对锁紧机构中的卡瓦体零件进行分析与对比, 确保该系统能够满足设计要 求。首先建立4组卡瓦体模型, 以A、B、C、D表示。四种卡瓦体模型仅在棱带倾角、过盈量 方面有差别, 具体参数见表1。

表 1 不同卡瓦体模型的结构参数

\begin{tabular}{|c|c|c|c|c|c|}
\hline 扇形角度 ${ }^{\circ}$ & 棱带高度 $/ \mathrm{mm}$ & 高度 $/ \mathrm{mm}$ & 棱带倾角 $/{ }^{\circ}$ & 过盈量 $/ \mathrm{mm}$ & 有限元模型 \\
\hline 36 & 3 & 36 & 15 & 0.05 & $\mathrm{~A}$ \\
\hline 36 & 3 & 36 & 15 & 0.03 & $\mathrm{~B}$ \\
\hline 36 & 3 & 36 & 12 & 0.03 & $\mathrm{C}$ \\
\hline 36 & 3 & 36 & 10 & 0.03 & $\mathrm{D}$ \\
\hline
\end{tabular}




\section{1 过盈量和棱带倾角与强度的关系}

利用有限元软件Ansys对卡瓦体模型进行几何简化、网格划分、接触设置以及约束施加后, 得到四种模型的最大Mises应力 $\sigma_{M}$ 的变化曲线, 如图6。

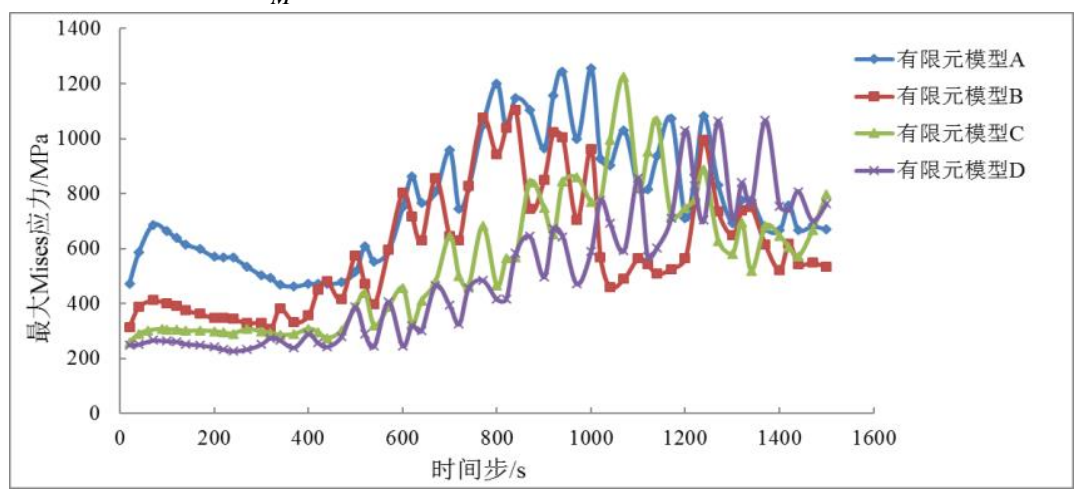

图6 锁紧机构不同卡瓦体模型的最大Mises应力变化曲线

从图 6 中可以看到: 在 $1000 \mathrm{~s}$ 之前, $\sigma_{M}$ 呈波动增加的趋势, 并且四种模型的 $\sigma_{M}$ 大小顺 序为: $\mathrm{A}>\mathrm{B}>\mathrm{C}>\mathrm{D}$ 。模型 $\mathrm{A} 、 \mathrm{~B} 、 \mathrm{C} 、 \mathrm{D}$ 的最大 Mises 应力 $\sigma_{M}$ 分别为 $1254.9 \mathrm{MPa} 、 1104.2 \mathrm{MPa}$ 、 1223.5 MPa、1064.2 MPa。紧锁机构的卡瓦体材料使用 60Si2CrVA 钛合金, 该材料屈服强度 $\sigma_{s}$ 为 $1667 \mathrm{MPa}^{[7]}$ 。以卡瓦的屈服强度 $\sigma_{s}$ 为极限应力, 根据公式:

$$
n=\frac{\sigma_{s}}{\sigma_{M}}
$$

式中: $n$ 为安全系数, $\sigma_{s}$ 为卡瓦材料屈服强度, $\sigma_{M}$ 为最大 Mises 应力。

计算出模型 $\mathrm{A} 、 \mathrm{~B} 、 \mathrm{C} 、 \mathrm{D}$ 的安全系数 $n$ 分别为 $1.32 、 1.50 、 1.36 、 1.56$ 。所以减小过盈量 和棱带倾角可以降低 $\sigma_{M}$, 提高卡瓦体强度。并且改变卡瓦体的过盈量和棱带倾角, 其安全系 数变化范围小, 均满足安全要求。

\section{2 过盈量与棱带倾角与释放力的关系}

四种卡瓦体模型轴向作用力 $F_{y}$ 的变化曲线如图 7 所示。从图 7 中可以得到: 在时间 1000 $\mathrm{s}$ 之前, $F_{y}$ 呈平稳变化趋势, 并且四种模型 $F_{y}$ 的大小排序为, $\mathrm{A}>\mathrm{B}>\mathrm{C}>\mathrm{D} 。 \mathrm{~A} 、 \mathrm{~B} 、 \mathrm{C} 、 \mathrm{D}$ 的最 大轴向作用力 $F_{y}$ 分别为 $1723.1 \mathrm{~N} 、 1048.5 \mathrm{~N} 、 922.6 \mathrm{~N} 、 790.3 \mathrm{~N}$ 。可以得到锁紧机构模型的释 放力 $F_{s}$ 的计算公式:

$$
F_{s}=F_{y} \times 24 \times 2
$$

式中: $F_{s}$ 为锁紧机构释放力, $F_{y}$ 为最大轴向作用力。通过表 2 可以看出改变卡瓦体的过 盈量和棱带倾角, $F_{y}$ 变化范围大。改变卡瓦体过盈量及棱带倾角, 锁紧机构零件强度变化稳 定, 释放力变化较大。因此锁紧机构在满足强度设计要求的前提下, 可以在较大的范围内调 节释放力。

表 2 不同卡瓦体模型有限元分析数据

\begin{tabular}{|c|c|c|c|c|c|}
\hline 有限元模型 & $\sigma_{\mathrm{s}} / \mathrm{MPa}$ & $\sigma_{\mathrm{M}} / \mathrm{MPa}$ & $n$ & $F_{\mathrm{y}} / \mathrm{MPa}$ & $F_{\mathrm{s}} / \mathrm{kN}$ \\
\hline $\mathrm{A}$ & 1667 & 1254.9 & 1.32 & 1723.1 & 82.71 \\
\hline $\mathrm{B}$ & 1667 & 1104.2 & 1.50 & 1048.5 & 50.33 \\
\hline $\mathrm{C}$ & 1667 & 1223.5 & 1.36 & 922.57 & 44.28 \\
\hline $\mathrm{D}$ & 1667 & 1064.2 & 1.56 & 790.31 & 37.94 \\
\hline
\end{tabular}




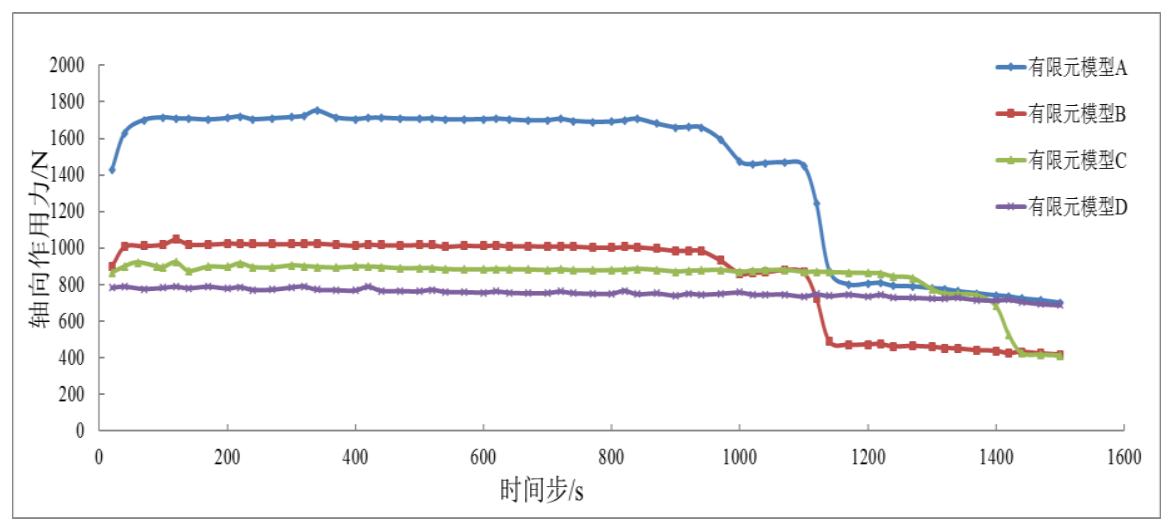

图 7 不同卡瓦体模型的轴向作用力变化曲线

\section{4. 井下机械震源可靠性分析}

井下机械震源在工作过程中, 要承受上部钻具施加的巨大压力还要面对井下高温的工作 环境, 长时间工作可能会发生故障。一旦发生故障正常的钻井作业就会受到影响。因此在设 计过程中要对可能发生的潜在故障进行分析, 提出相应的改善控制措施。

\section{1 震源失效故障树建立}

设计的井下机械震源的主体是筒体组件, 在工作时, 承受了拉、压、弯等作用力; 密封 组件也承担着防止钻井液的进入、润滑油的泄露的作用 ${ }^{[9]}$ 。这两部分组件在震源不断地重复 工作中, 发生故障的概率最大。其中, 筒体组件最大的潜在故障模式是筒体组件断裂失效和 裂纹失效; 密封组件最大的故障模式是腐蚀失效。这些潜在故障的发生均有可能导致震源系 统的功能失效。

以 “井下震源失效” 为顶事件, 建立震源失效的故障树系统图, 如图8。引起井下震源失 效的直接原因有: 筒体组件断裂失效、筒体组件裂纹失效以及密封组件腐蚀失效。以这三种 故障模式为顶事件建立引起井下震源失效的故障树, 如图9。找到引起井下震源失效的共因事 件，包括结构应力集中、材料㓞性不足。

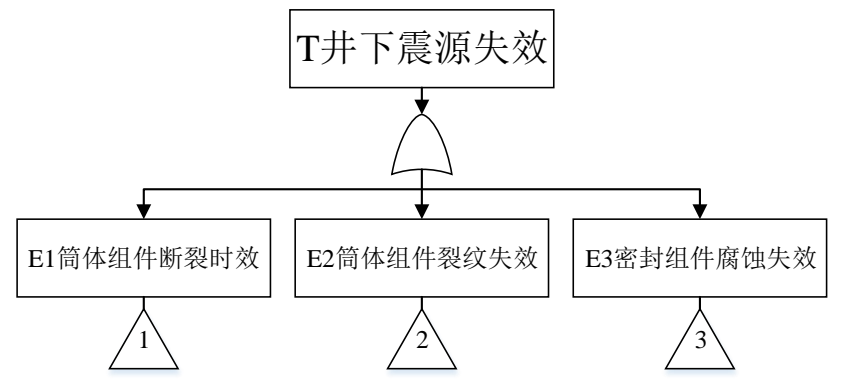

图8 井下震源失效二级故障树 

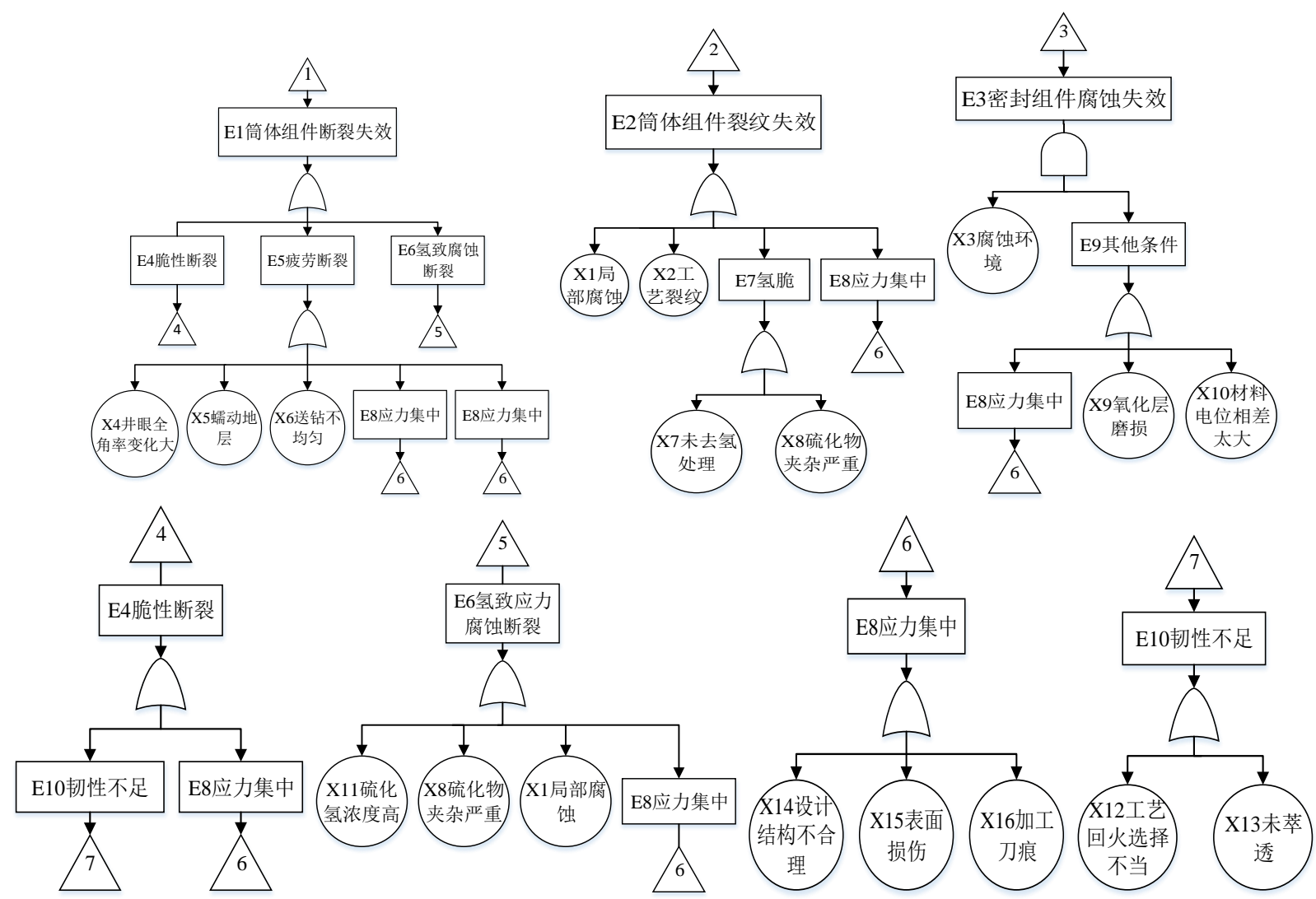

图9引起井下震源失效的三种故障模式故障树

\section{2 失效模式分析及相应控制措施}

利用下行法对故障树进行分析, 可以得到 “井下震源失效” 故障树的最小割集有 16 个, 为: $\{X 1\},\{X 2\},\{X 4\},\{X 5\},\{X 6\},\{X 7\},\{X 8\},\{X 11\},\{X 12\},\{X 13\},\{X 14\},\{X 15\}$, $\{X 16\},\{X 13, X 14, X 15, X 16\},\{X 3, X 9\},\{X 3, X 10\}$ 。二阶和四阶最小割集发生的概率较小, 一阶最小割集发生的概率相对较大。应重点针对底层共因事件提出相应改善控制措施。

主要通过在加工制作时采取以下措施进行控制:

（1）在加工时选择韧性高、耐腐蚀、抗氢脆的材料, 以降低疲劳裂纹产生的可能性, 从 而提高机械震源的寿命;

（2）改进热处理工艺，提高材料的韧性;

（3）涂层防护，提高机械震源的抗腐蚀性能;

（4）加强使用管理严格按照规范使用, 避免在使用时造成震源机械损伤。

表3 下行法分析震源失效故障树结果

\begin{tabular}{|c|c|c|c|c|c|c|c|}
\hline 第一级 & 第二级 & 第三级 & \multicolumn{2}{|c|}{ 第四级 } & \multicolumn{3}{|c|}{ 第五级 } \\
\hline $\mathrm{T}$ & $\mathrm{E} 1$ & $\mathrm{E} 4$ & $\mathrm{E} 10$ & $\mathrm{X} 1$ & $\mathrm{X} 12$ & $\mathrm{X} 1$ & $\mathrm{X} 13$ \\
\hline & $\mathrm{E} 2$ & $\mathrm{E} 5$ & $\mathrm{E} 8$ & $\mathrm{X} 2$ & $\mathrm{X} 13$ & $\mathrm{X} 2$ & $\mathrm{X} 11$ \\
\hline & $\mathrm{E} 3$ & $\mathrm{E} 6$ & $\mathrm{X} 4$ & $\mathrm{X} 7$ & $\mathrm{X} 14$ & $\mathrm{X} 7$ & $\mathrm{X} 8$ \\
\hline & & $\mathrm{X} 1$ & $\mathrm{X} 5$ & $\mathrm{X} 8$ & $\mathrm{X} 15$ & $\mathrm{X} 8$ & $\mathrm{X} 15$ \\
\hline & & $\mathrm{E} 7$ & $\mathrm{E} 8$ & $\mathrm{X} 15$ & $\mathrm{X} 4$ & $\mathrm{X} 3, \mathrm{X} 14$ & \\
\hline & & $\mathrm{E} 8$ & $\mathrm{E} 10$ & $\mathrm{X} 16$ & $\mathrm{X} 5$ & $\mathrm{X} 3, \mathrm{X} 16$ & \\
\hline & & $\mathrm{X} 3, \mathrm{E} 9$ & $\mathrm{X} 11$ & $\mathrm{X} 3, \mathrm{E} 8$ & $\mathrm{X} 6$ & $\mathrm{X} 3, \mathrm{X} 10$ & \\
\hline & & & $\mathrm{X} 3, \mathrm{X} 10$ & $\mathrm{X} 3, \mathrm{X} 9$ & $\mathrm{X} 14$ & $\mathrm{X} 3, \mathrm{X} 14$ & \\
& & & & & & & \\
\hline
\end{tabular}




\section{5. 结论}

（1）设计了一种井下机械震源，包括：下击装置、上提装置、扭矩传递装置及密封装置。

（2）使用有限元软件，对震源锁紧机构进行技术分析，得到卡瓦体参数与机构强度及释 放力之间的关系，验证本文设计的锁紧机构能够大范围调节释放力。

（3）通过对潜在故障模式的分析, 确定筒体组件断裂失效、密封组件腐蚀失效及筒体组 件裂纹失效三种故障模式。建立 “井下震源失效” 的故障树, 提出减少震源失效概率的措施。

\section{致谢}

本文为国家 “973” 计划项目 “深水钻完井工程风险预警及管控” (2015CB251200)和 “十 二五” 国家科技重大专项 “加密调整井网防碰示范工程” (2011ZX05057-002-006)的成果之一。

\section{References}

[1] Gang LIU, Xiangyang XIA, Chuang LIU, et al. Application of real-time monitoring technology of wellbore anti-collision in a well in Bohai. Science Technology and Engineering, vol. 15,pp. 123-126, 2015.

[2] Gang LIU, Jin SUN, Baosheng HE, et al. Design and field test of surface monitoring system for directional wells anti-collision. Petroleum Drilling Techniques, vol. 1,pp. 7-11, 2012.

[3] Yinao SU, Yi XU, Limin SHENG, et al. Review on study progress of seismic while drilling technology. Oil Drilling \& Production Techniques, vol. 5,pp. 1-7, 2010.

[4] Rector J W. The use of drill-bit energy as a downhole seismic source. Geophysics, vol. 56, pp. 628, 1991.

[5] Jun XIE, Le LI, Wenfeng LIU. Selection of wavelet basis in noise elimination of vibration signals. Science. Technology and Engineering, vol. 11,pp. 5997-6000, 2011.

[6] Jiaqi ZHOU, Guotian ZHANG, Dongyang LI, et al. Development and application of full hydraulic type drilling Rig. China Petroleum Machinery, vol. 9,pp. 89-91, 2009.

[7] Yuxian ZHANG, Hong WANG. Laboratorial research on yield strength of materials. Journal of Materials Engineering, vol. 11,pp. 43-45, 2005.

[8] Xuefei WANG, Qing LI, Li FENG. Aircraft fault diagnosis method based on model and fault-tree. Science Technology and Engineering, vol. 17,pp. 308-313, 2017.

[9] Jinbiao LIU, Zhiyong TONG. Leakage and prevention of sealing device. Hydraulics Pneumatics \& Seals, vol. 31,pp. 41-42, 2011. 